\title{
氮磷共掺杂一提升氧还原催化剂毒化分子耐受性 $\left(\mathrm{SO}_{x}, \mathrm{NO}_{x}\right.$ 和 $\left.\mathrm{PO}_{x}\right)$
}

庄林

武汉大学化学与分子工程学院, 武汉 430072

\section{$\mathrm{P}, \mathrm{N}$-Doped Carbon as an Efficient Anti-Poisoning Catalyst Against $\mathrm{SO}_{\boldsymbol{x}}$, $\mathrm{NO}_{x}$ and $\mathrm{PO}_{x}$ during Oxygen Reduction in Acidic Media}

ZHUANG Lin

School of Chemistry and Molecular Engineering, Wuhan University, Wuhan 430072, P. R. China.

Email: lzhuang@whu.edu.cn.

Published online: September 27, 2018.

(a)

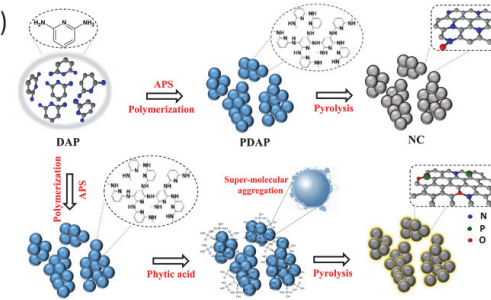

(b)
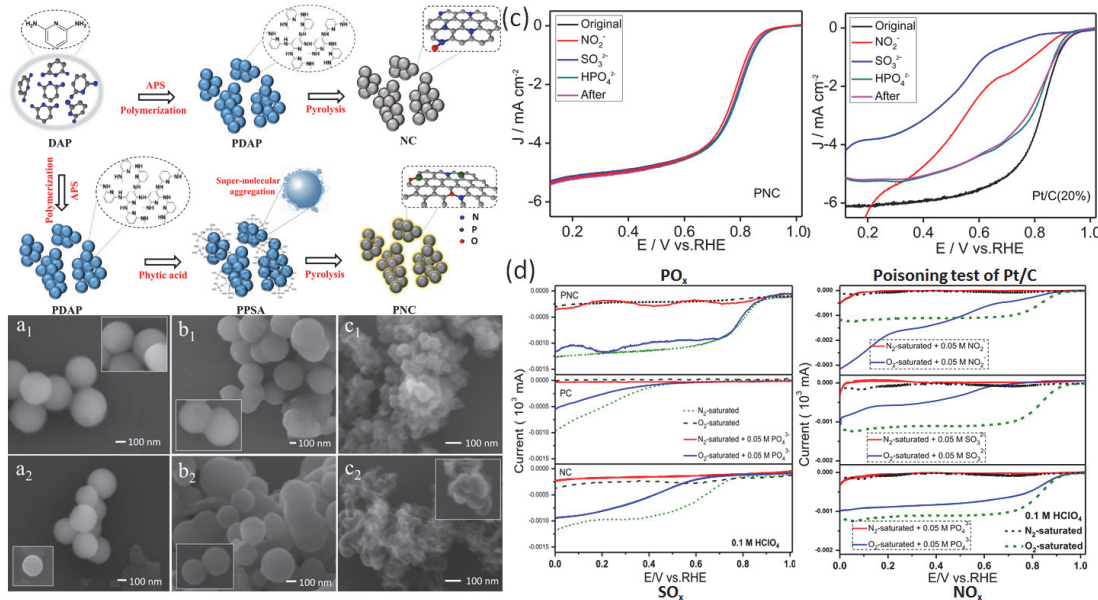

(e)
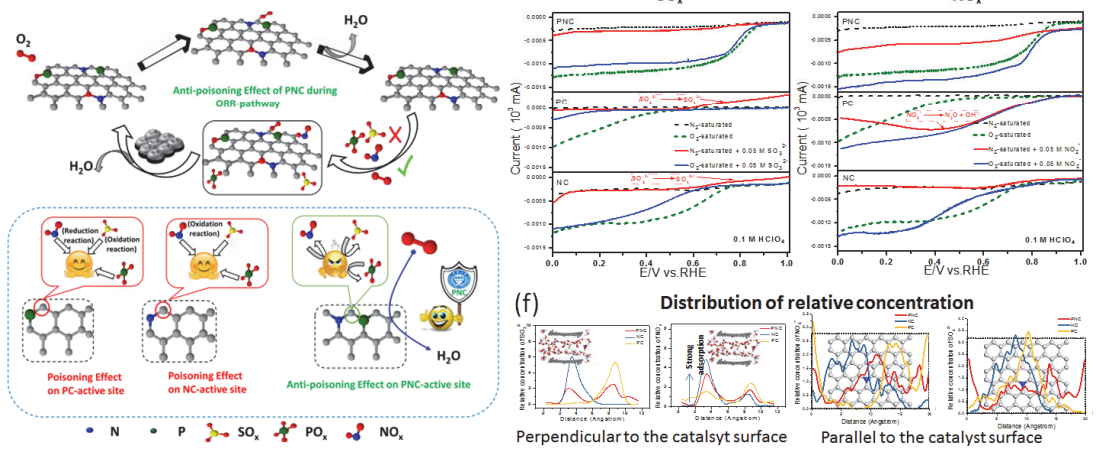

(a) PNC 催化剂合成示意图。(b) PNG, NC 和 PC 扫描电镜。(c) PNC 和 Pt/C 催化剂抗中毒性能测试(背景电流扣除)。

(d) PNC, PC, NC 和 Pt/C 催化剂毒化环境电化学性能。(e) PNC 抗中毒性能示意图。

(f) 分子动力学模拟催化表面毒化分子浓度分布。

燃料电池以氢气为燃料, 以空气为氧化剂。在 电催化剂的作用下, 电池阳极和阴极分别发生氢
氧化反应和氧还原反应 (Oxygen reduction reaction, ORR)。在接触空气的阴极氧还原的过程 
中, 即使空气中存在少量的硫氧化物 $\left(\mathrm{SO}_{x}\right)$ 或氮氧 化物 $\left(\mathrm{NO}_{x}\right)$, 也可轻易毒化包括 $\mathrm{Pt}$ 基催化剂, 极大降 低催化剂的活性, 进而损害燃料电池的耐久性 ${ }^{1-4}$ 。 此外, 以磷酸掺杂的聚苯咪唑为电解质的新型高 温燃料电池, 磷酸根 $\mathrm{PO}_{4}^{3-}$ 的流失不仅会导致电解 质膜的性能衰减, 还会导致催化剂的磷酸根毒化 吸附, 降低高温燃料电池的性能和耐久性 5,6 。

因此, 开发具有高活性和稳定性的燃料电池 抗中毒催化剂显得尤为重要。

最近, 重庆大学化学化工学院魏子栋教授团 队研究发现: 氮磷共掺杂碳催化剂(PNC)具有抗 $\mathrm{SO}_{x} 、 \mathrm{NO}_{x}$ 和 $\mathrm{PO}_{x}$ 中毒特性。研究者根据他们稍早一 些的理论计算, 发现: 相比氮、磷、嗍对碳的单掺 杂, 这些元素的共掺杂, 使 $\mathrm{O}_{2}$ 分子有单端吸附转变 为两端吸附, 更加有利于活化, $\mathrm{O}-\mathrm{O}$ 键解离活化 能从 $1.8 \mathrm{eV}$ 下降为 $0.5 \mathrm{eV}^{7,8}$ 。他们采用过硫酸铵引 发二胺吡啶聚合成的聚二胺吡啶(PDAP)为氮碳前 驱体, 通过氢键在PDAP表面原位自组装的植酸超 分子集合体 (PPSA)为磷源, 直接热解合成 $N$ 、 $\mathrm{P}$ 共 掺杂多孔碳材料催化剂。他们意外地发现: PNC 催化剂在酸性介质中不仅具有优异的ORR催化活 性, 而且具有优异的抗 $\mathrm{SO}_{x} 、 \mathrm{NO}_{x}$ 和 $\mathrm{PO}_{x}$ 毒化作用, 其ORR催化性质几乎不受毒物的影响。相同条件 下, $\mathrm{Pt} / \mathrm{C}$ 、氮或磷单掺杂的 $\mathrm{NC}$ 或 $\mathrm{PC}$ 都不具备抗中 毒性质, 催化剂呈现不可逆的活性衰减。

PPSA的引入实现了氮碳材料表面原位掺P和 表面碳缺陷结构构筑, 诱导并促进 $\mathrm{N}$ 杂原子掺入碳 环结构, 形成 $\mathrm{N} / \mathrm{P}$ 原子比为 $1: 1$ 的 $\mathrm{P}-\mathrm{N}$ 共掺杂结构。 但是, 磷源的过量使用会破坏原有碳环结构, 使 $\mathrm{N}$ 由6-ring N掺杂结构(Pyridinic-N、Graphitic-N)) 向 5ring $\mathrm{N}$ 掺杂结构 $($ Pyrrolic- $\mathrm{N}$ )转换, 而 $\mathrm{P}$ 原子本身则 会更倾向结合 $\mathrm{O}$, 形成P-O而非P-C掺杂结构, 导致 $\mathrm{P}-\mathrm{N}$ 共掺杂结构的破坏, 最终导致活性和抗中毒性 质的下降。

$\mathrm{N}$ 和P共同掺杂结构结构会使得邻近的碳原子 的电子密度重新分配, 强化氧气在催化剂表面的 选择性吸附。分子动力学模拟表明: 相比 $\mathrm{P}$ 或 $\mathrm{N}$ 单 杂原子掺杂结构, P-N共掺杂的结构可显著降低 $\mathrm{NO}_{x}$ 和 $\mathrm{SO}_{x}$ 在催化剂表面的富集程度, 抑制了 $\mathrm{NO}_{x}$
在催化剂活性中心的毒化吸附, 消除 $\mathrm{SO}_{x}$ 分子在催 化剂表面的聚集。而在 $\mathrm{NC}$ 和 $\mathrm{PC}$ 催化剂表面, $\mathrm{NO}_{x}$ 倾向吸附在杂原子临近的 $\mathrm{C}$ 位点 $(\mathrm{ORR}$ 的主要活性 位), $\mathrm{SO}_{x}$ 则倾向大量聚集在表面所有位点, 造成催 化剂中毒。

研究中采用植酸诱导形成 $\mathrm{N} / \mathrm{P}$ 原子比为 $1: 1$ 的 共掺杂结构, 具有显著的抗中毒能力; 而当过量植 酸引入会破坏N-P共掺杂结构, 则导致PNC催化剂 活性下降和抗中毒能力下降。

该工作近期以 “An Efficient Anti-Poisoning Catalyst Against $\mathrm{SO}_{x}, \mathrm{NO}_{x}$ and $\mathrm{PO}_{x}$ : $\mathrm{P}, \mathrm{N}$-Doped Carbon for Oxygen Reduction in Acidic Media” 为 题在线发表在Angewandte Chemie International Edition 上。 $^{9}$

研究首次揭示了 P-N共掺杂结构的抗中毒特 性, 成功研制了具有抗 $\mathrm{SO}_{x} 、 \mathrm{NO}_{x}$ 和 $\mathrm{PO}_{x}$ 中毒的高活 性PNC催化剂, 开创了抗中毒的燃料电池ORR催 化剂设计新途径。

\section{References}

(1) Chen, Z.; Higgins, D.; Yu, A.; Zhang, L.; Zhang, J. Energy Environ. Sci. 2011, 4, 3167. doi: 10.1039/c0ee00558d

(2) Zhai, Y.; Bender, G.; Dorn, S.; Rocheleau, R. J. Electrochem. Soc. 2010, 157, B20. doi: 10.1149/1.3247546

(3) Zhang, Q.; Mamtani, K.; Jain, D.; Ozkan, U.; Asthagiri, A. J. Phys. Chem. C 2016, 120, 15173. doi: 10.1021/acs.jpcc.6b03933

(4) Zhang, P.; Chen, X. F.; Lian, J. S.; Jiang, Q. J. Phys. Chem. C 2012, 116, 17572. doi: 10.1021/jp304097m

(5) He, Q.; Shyam, B.; Nishijima, M.; Ramaker, D.; Mukerjee, S. J. Phys. Chem. C 2013, 117, 4877. doi: 10.1021/jp309282n

(6) Li, Q.; Wu, G.; Cullen, D. A.; More, K. L.; Mack, N. H.; Chung, H. T.; Zelenay, P. ACS Catal. 2014, 4, 3193. doi: 10.1021/cs500807v

(7) Li, R.; Wei, Z.; Gou, X. ACS Catal. 2015, 5, 4133. doi: 10.1021/acscatal.5b00601

(8) Yang, N.; Li, L.; Li, J.; Ding, W.; Wei, Z. Chem. Sci. 2018, 9, 5795. doi: $10.1039 / \mathrm{c} 8 \mathrm{sc} 01801 \mathrm{~d}$

(9) Najam, T.; Shah, S. A.; Ding, W.; Jiang, J.; Li, J.; Wang, Y.; Li, L.; Wei, Z. Angew. Chem. Int. Ed. 2018, doi: 10.1002/anie.201808383 